\title{
Current Curative Surgical Management of Gallbladder Cancer: A Brief Review
}

\author{
Theodoros E Pavlidis ${ }^{\mathrm{a}, \mathrm{b}}$, Efstathios T Pavlidis ${ }^{\mathrm{a}}$, Nikolaos G Symeonidis ${ }^{\mathrm{a}}$, \\ Kyriakos Psarras ${ }^{\text {a }}$, Athanasios K Sakantamis ${ }^{\mathrm{a}}$
}

\begin{abstract}
Gallbladder cancer has a dismal prognosis, radical surgical resection offers the only chance of cure, but it is possible in only small proportion due to advanced disease and the recurrence is high. The extent of resection depends on the extent of disease. The aim of any therapeutic operation must be the radical removal of the tumor with no residual disease (R0 resection). Crucial for the surgical strategy is the TNM staging and the planning is decided in accordance with the $\mathrm{T}$ stage. In T1a simple cholecystectomy is considered as an adequate treatment. In T1b there is a controversy; some advocate simple cholecystectomy and others more extensive radical cholecystectomy. In T2 either extended radical cholecystectomy or resection of liver segments IVb and V. In all cases a radical lymph node dissection along the hepatoduodenal ligament is needed additionally. In T3 either hepatic segmentectomy IVb and V at least or extended right hepatectomy, both combined always with radical lymph node dissection. In T4 usually palliative operation; an extensive en-block resection with removal of included organs is rarely attempted.
\end{abstract}

Keywords: Gallbladder cancer; Biliary tree tumors; Radical cholecystectomy; Extrahepatic; Cholangiocarcinoma; Biliary diseases

\section{Review}

Gallbladder carcinoma although rare, is the most common and most aggressive cancer of the biliary tree; it is one of the most highly malignant tumors with poor prognosis and still poses many challenges to the surgeon. It occurs more

Manuscript accepted for publication June 7, 2012

${ }^{a}$ Aristotle University of Thessaloniki, Medical School, Second Surgical Propedeutic Department, Hippocration Hospital, Konstantinoupoleos 49, 54642 Thessaloniki, Greece

${ }^{\mathrm{b}}$ Corresponding author: Theodoros E Pavlidis, A Samothraki 23, 54248

Thessaloniki, Greece. Email: pavlidth@auth.gr

doi: $10.4021 / \mathrm{jcs} 50 \mathrm{w}$ frequently in women (ratio $2.5-3 / 1$ ) and especially in those over 65 years and with long-lasting gallstones, while there is a frequent association with gene mutations, such as genes K-ras, p53 [1-4].

The gallstones are the main and most well-known risk factor for gallbladder cancer, since it coexists in $80-90 \%$ of cancer cases at diagnosis and $1-3 \%$ of patients with gallstones develop cancer. Large gallstones cause injury to the gallbladder mucosa and chronic inflammation, which can lead to atypia, dysplasia and cancer development over time at least $10-15$ years. The other risk factors include porcelain gallbladder - mainly focal microcalcification in the mucosa than in the wall, the abnormal junction of pancreatic and bile duct causing reflux of pancreatic fluid and toxic effect on the mucosa by activation of pancreatic enzymes, polyps of the gallbladder especially those with size greater than $10 \mathrm{~mm}$, the xanthogranulomatous cholecystitis, chronic typhoid infection, gallbladder adenomyomatosis and inflammatory bowel disease [1].

The location affects mainly the fundus (60\%), body $(30 \%)$ and neck $(10 \%)$. The diagnosis is based on modern imaging ultrasound, computed tomography and magnetic resonance imaging, which is the method of choice as the most pathognomonic tool. Recently, pozitron emission tomography (PET-FDG) has been used in selected cases of small-occult questionable primary lesion or detection of local residual disease after cholecystectomy as well as latent distant metastases. Preoperative staging of the disease is achieved by these imaging tests and determines the strategy of surgical treatment $[3,5]$. The staging by TNM is the most used reliable classification. The $\mathrm{T}$ corresponds to the depth of the primary tumor infiltration and local extension. Thus, in Tis the lesion confined to the epithelium, while it invades in T1a the lamina propria, in T1b the muscle layer, in T2 the perimuscular connective tissue, in T3 the serosa or directly the liver or adjacent organs (stomach, duodenum, colon, pancreas, great omentum, common bile duct) and in T4 the portal vein, hepatic artery or multiple extrahepatic organs. In N0 there are not infiltrated regional lymph nodes and in M0 there are no distant metastases.

The extent of resection depends on the extent of disease. The aim of any therapeutic operation must be the radical re- 
moval of the tumor with no residual disease (R0 resection). Crucial for the surgical strategy is the TNM staging and the planning is decided in accordance with the T stage $[3,5-8]$.

So, in T1a simple cholecystectomy is considered as an adequate treatment. In T1b there is a controversy; some advocate simple cholecystectomy and others more extensive radical cholecystectomy. The NCCN current guidelines recommend simple cholecystectomy for T1a lesions and radical cholecystectomy for T1b ones [3].

In $\mathrm{T} 2$ either extended radical cholecystectomy (wedge resection of the gallbladder bed with removal of liver parenchyma at least $2 \mathrm{~cm}$ ) or resection of liver segments $\mathrm{IVb}$ and $\mathrm{V}$. In all cases a radical lymph node dissection along the hepatoduodenal ligament is needed additionally.

In $\mathrm{T} 3$ either hepatic segmentectomy $\mathrm{IVb}$ and $\mathrm{V}$ at least or extended right hepatectomy, both combined always with radical lymph node dissection.

In T4 usually palliative operation; an extensive en-block resection with removal of included organs is rarely attempted. Recent studies from Japan have recommended aggressive surgery in T4 in selected patients, with extensive resections including right hepatectomy combined with pancreatoduodenectomy or resection of great vessels and restoration. It has been reported a rate of resectability $65.8 \%$, mortality $11.4 \%$ and five-year survival $13.7 \%$ [9]. Even the presence of positive paraaortic lymph nodes is not considered as an absolute contraindication; but the decision should better be individualized [10]. The role of chemotherapy in this advanced stage has been investigated with the administration of gemcitabine mainly, but also platinum or 5-FU derivatives. It has been reported a rate of response $24 \%$, median disease-free survival 4.1 months and median overall survival 7.5 months [11].

In general in gallbladder cancer, the role of the radiation, chemotherapy or even combination of both, either neoadjuvant or adjuvant as well as the current target therapy is limited and it remains to be determined in prospective randomized studies.

Diagnostic laparoscopy can prevent unnecessary laparotomy by identifying occult peritoneal metastasis in approximately $50 \%$. Therefore, its routine application is nowadays recommended. Also, the routine performance of lymph node dissection is mandatory (hepatoduodenal ligament, portal vein, retroduodenal). The exception is only T1a lesion incidentally discovered after previous cholecystectomy.

In about half of patients with gallbladder cancer, the diagnosis is established randomly by the pathologist after cholecystectomy. Early reoperation is strongly recommended in stage $\mathrm{T} 2$, because it has a positive effect on survival $[12,13]$. It also has a place in stage $\mathrm{T} 3$ due to the high rate of positive lymph nodes, although it has not been found any advantage in survival $[12,14]$. However, another study after laparoscopic cholecystectomy and incidental discover of gallbladder cancer $(0.28 \%)$ found significant benefit in survival in stage $\mathrm{T} 2$ and $\mathrm{T} 3 \mathrm{after}$ reoperation for extensive resection of the gallbladder bed and lymph node dissection [15].

It is of great significance the state of cystic duct in the specimen of prior cholecystectomy. Its infiltration is associated with poorer prognosis and then it is additional necessary to the above mentioned, the bile duct resection with Rouxen Y hepaticojejunostomy. If it is not infiltrated, only lymph node dissection and radical removal of the gallbladder bed will be required [3].

Despite the above mentioned, a recent large nationwide statistical analysis from the USA included 2,955 patients with gallbladder cancer, found that only in $13 \%$ a radical resection / hepatectomy has been performed, with median survival of 16 months and five-year survival of $21.3 \%$ [16].

Thus, gallbladder cancer has a dismal prognosis, radical surgical resection offers the only chance of cure, but it is possible in only small proportion due to advanced disease and the recurrence is high.

\section{Funding}

None.

\section{Ethical Approval}

Not needed.

\section{Competing Interest}

No benefits in any form have been received or will be received from a commercial party related directly or indirectly to the subject of this article.

\section{References}

1. Reid KM, Ramos-De la Medina A, Donohue JH. Diagnosis and surgical management of gallbladder cancer: a review. J Gastrointest Surg. 2007;11(5):671-681.

2. Miller G, Jarnagin WR. Gallbladder carcinoma. Eur J Surg Oncol. 2008;34(3):306-312.

3. Zhu AX, Hong TS, Hezel AF, Kooby DA. Current management of gallbladder carcinoma. Oncologist. 2010;15(2):168-181.

4. Grobmyer SR, Lieberman MD, Daly JM. Gallbladder cancer in the twentieth century: single institution's experience. World J Surg. 2004;28(1):47-49.

5. Pilgrim C, Usatoff V, Evans PM. A review of the surgical strategies for the management of gallbladder carcinoma based on $\mathrm{T}$ stage and growth type of the tumour. Eur J Surg Oncol. 2009;35(9):903-907.

6. Foster JM, Hoshi H, Gibbs JF, Iyer R, Javle M, Chu Q, 
Kuvshinoff B. Gallbladder cancer: Defining the indications for primary radical resection and radical re-resection. Ann Surg Oncol. 2007;14(2):833-840.

7. Mekeel KL, Hemming AW. Surgical management of gallbladder carcinoma: a review. J Gastrointest Surg. 2007;11(9):1188-1193.

8. Shih SP, Schulick RD, Cameron JL, Lillemoe KD, Pitt HA, Choti MA, Campbell KA, et al. Gallbladder cancer: the role of laparoscopy and radical resection. Ann Surg. 2007;245(6):893-901.

9. Shimizu H, Kimura F, Yoshidome H, Ohtsuka M, Kato A, Yoshitomi H, Nozawa S, et al. Aggressive surgical approach for stage IV gallbladder carcinoma based on Japanese Society of Biliary Surgery classification. J Hepatobiliary Pancreat Surg. 2007;14(4):358-365.

10. Nishio H, Nagino M, Ebata T, Yokoyama Y, Igami T, Nimura Y. Aggressive surgery for stage IV gallbladder carcinoma; what are the contraindications? J Hepatobiliary Pancreat Surg. 2007;14(4):351-357.

11. Cassier PA, Thevenet C, Walter T, Baulieux J, Scoazec JY, Bancel B, Adham M, et al. Outcome of patients receiving chemotherapy for advanced biliary tract or gallbladder carcinoma. Eur J Gastroenterol Hepatol.
2010;22(9):1111-1117.

12. Goetze TO, Paolucci V. Benefits of reoperation of T2 and more advanced incidental gallbladder carcinoma: analysis of the German registry. Ann Surg. 2008;247(1):104108.

13. Wakai T, Shirai Y, Hatakeyama K. Radical second resection provides survival benefit for patients with T2 gallbladder carcinoma first discovered after laparoscopic cholecystectomy. World J Surg. 2002;26(7):867-871.

14. Goetze TO, Paolucci V. Adequate extent in radical reresection of incidental gallbladder carcinoma: analysis of the German Registry. Surg Endosc. 2010;24(9):21562164.

15. Glauser PM, Strub D, Kaser SA, Mattiello D, Rieben F, Maurer CA. Incidence, management, and outcome of incidental gallbladder carcinoma: analysis of the database of the Swiss association of laparoscopic and thoracoscopic surgery. Surg Endosc. 2010;24(9):2281-2286.

16. Mayo SC, Shore AD, Nathan H, Edil B, Wolfgang CL, Hirose K, Herman J, et al. National trends in the management and survival of surgically managed gallbladder adenocarcinoma over 15 years: a population-based analysis. J Gastrointest Surg. 2010;14(10):1578-1591. 